A D VA N C E P H O T O N S O U C E

A R G O N E N A T I O N A L A B OR A T O R Y

\title{
LINAC-AUGMENTED LIGHT SOURCES
}

\author{
AN INCREMENTAL CONCEPT FOR \\ ENHANCING THE CAPABILITIES OF \\ EXISTING $3^{\text {RD }}-$ GENERATION STORAGE \\ RINGS
}

John W. Lewellen 


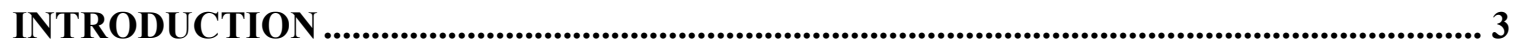

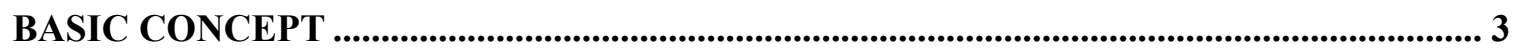

StORAGE RING PERFORMANCE ENHANCEMENTS ............................................................... 3

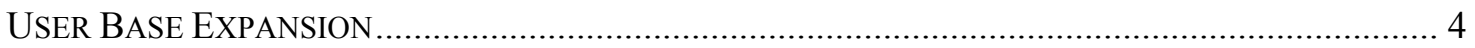

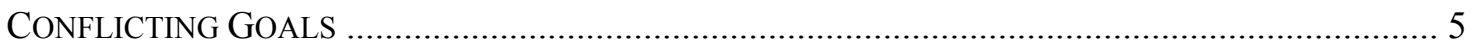

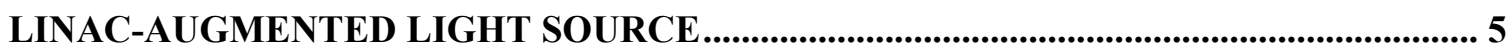

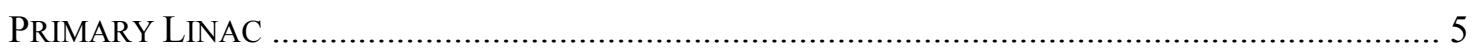

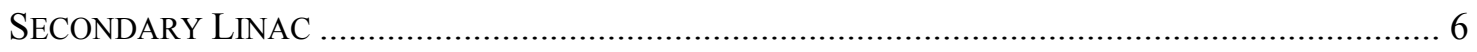

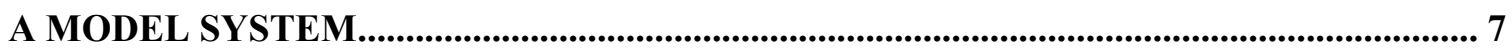

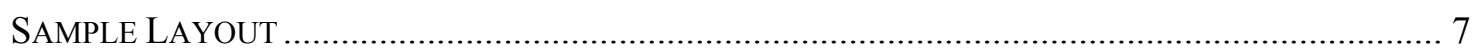

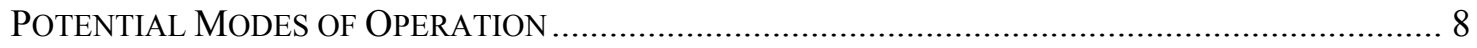

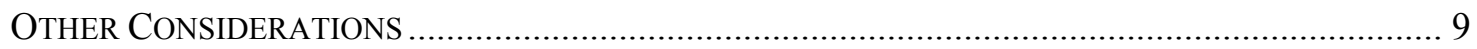

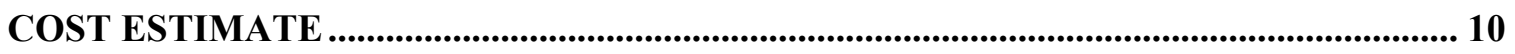

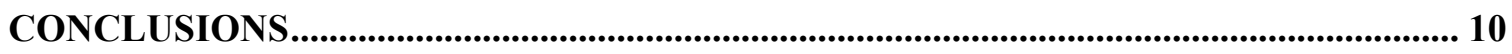

APPENDIX A: STORED BEAM LIMIT CALCULATIONS ............................................. 12

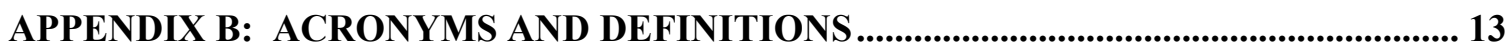

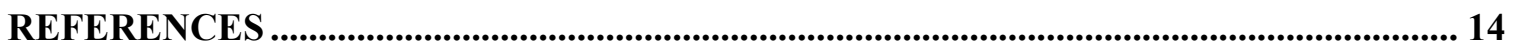




\title{
LINAC-AUGMENTED LIGHT SOURCES
}

\author{
AN INCREMENTAL CONCEPT FOR ENHANCING THE CAPABILITIES OF \\ EXISTING 3 RD_GENERATION STORAGE RINGS
}

\section{INTRODUCTION}

Planned and proposed $4^{\text {th }}$-generation $\mathrm{x}$-ray sources, such as energy-recovery linacs (ERLs) and single-pass $\mathrm{x}$-ray free-electron lasers (X-FELs) offer a number of potential advantages, including small source size, higher peak brightness, ultrashort pulses, and potentially temporally and transversely coherent pulses. While offering unique capabilities, such facilities will also offer several important limitations, including limited numbers of user beamlines (for FELs) and a pulse-repetition rate that may be too high for many dynamics experiments (ERLs). In addition, there are many technical challenges associated with both types of facilities.

A third type of facility, exemplified by the Short Pulse Photon Source (SPPS) at SLAC [1], would support neither a large number of users simultaneously nor generate coherent pulses, but would generate very intense, short $\mathrm{x}$-ray pulses. Such a facility could serve as the starting point for either an ERL or an X-FEL, or a combined, hybrid machine.

For the foreseeable future, however, existing $3^{\text {rd }}$-generation light source storage rings, such as the Advanced Photon Source, will continue to play important roles in supporting scientific research utilizing high-brightness $\mathrm{x}$-rays. Existing facilities offer the powerful combination of a large number of user beamlines, efficient use of electron beam energy, and established user communities, and a program of incremental investment in, and improvements to, these facilities should continue to pay dividends into the future. This document discusses potential upgrade paths based on the Advanced Photon Source (APS) as a model 3 rd-generation facility.

\section{BASIC CONCEPT}

If existing $3^{\text {rd }}$-generation facilities are to remain centers of excellence for light source-based research into the future, they must not only maintain and enhance their support of their existing user base, but also seek to expand their capabilities to support additional classes of users. There are several paths available toward this goal. The APS is already committed to providing enhanced technical and operational support to its resident users and beamlines, and this is expected to continue into the future. Given that this work will also include such topics as optimization of x-ray beamline optics and improvements in data acquisition software, additional gains in effective performance will be driven by the performance of the accelerator itself, and by additional capabilities which could be added to the APS accelerator complex.

\section{STORAGE RING PERFORMANCE ENHANCEMENTS}

The storage ring-related enhancements may be broken down into three basic categories. First, the devices that actually generate the radiation for the users may be enhanced; this includes the development and installation of new insertion devices such as superconducting small-gap or 
solenoid-derived undulators, yielding better source quality. Second, the transverse beam size (e.g., emittance and dispersion) may be reduced in the insertion devices, yielding smaller source sizes. Third, the stored beam current may be increased, perhaps in part with the installation of superconducting cavities, yielding more x-rays per unit time per insertion device. All of these paths are expected to be followed into the future, perhaps with additional enhancements such as beam sizes that can be varied sector by sector. These enhancements would benefit the current user base and should tend to draw in more $\mathrm{x}$-ray storage ring users.

Pushing the APS storage ring to higher beam currents and lower emittances will require refinement of the top-up technique, currently in use at the APS to maintain a stable stored-beam current. Refinements to the top-up methodology, such as booster pulse-stacking and "quiet" injections, may allow top-up to occur more often; however, the basic cycle rate of the APS booster limits it to $2-\mathrm{Hz}$ operation. With a notional injection of $5 \mathrm{nC}$ per top-up shot, stored-beam current would be limited to $1.5 \mathrm{~A}$ if $0.1 \%$ charge stability is to be maintained. At a $2-\mathrm{Hz}$ cycle rate, the lifetime could be no shorter than about 8 minutes if the $0.1 \%$ stability criterion is maintained. These are both very extreme conditions compared to the current operation of the APS (100 $\mathrm{mA}$ beam currents, topped off at 2-minute intervals, with lifetimes on the order of $5-20$ hours under normal operational conditions), however, it is entirely possible that operational conditions this extreme could be desired in the future. (A detailed discussion of issues relating to increasing the stored beam current significantly above $100 \mathrm{~mA}$, including safety envelope aspects for both existing and possible new machines, is beyond the scope of this paper.)

Both of these possibilities for the storage ring, however, assume the full availability of the current APS injection system, and the restrictions on that system translate directly into restrictions on ultimate performance of the storage ring. Pushing towards those limits would place severe limitations on the ability to transform other components of the APS injector system into user resources.

\section{USER BASE EXPANSION}

Expanding the user base to new classes of users, in contrast, could be accomplished via modifications to the APS injector complex. A first step in this direction has already been taken with the installation of the SPIRIT experiment in the APS Low-Energy Undulator Test Line (LEUTL) optical end station. The APS self-amplified spontaneous emission (SASE) FEL, currently installed in the LEUTL beam tunnel, has a demonstrated tuning range from $660 \mathrm{~nm}-130 \mathrm{~nm}$; the SPIRIT experiment makes use of the short wavelength range, tunability, and high peak optical pulse powers available. At the moment, the SPIRIT experiment can only be operated when the storage ring is not operating in top-up; however, this will be addressed if LEUTL is expanded to a true user facility. If this should occur, the APS linac will be playing a dual role, both as part of the storage ring injector complex and as the primary beam source for a first-generation SASE-FEL light source.

Along those lines, the APS injector complex contains two other machines that might be considered underutilized at the moment. With a lattice redesign, the booster synchrotron could, in theory, be converted into a UV-VUV storage ring capable of serving a number of users while preserving the ability to provide beam to the storage ring. By using a backscatter laser, the booster synchrotron could potentially also be used as a source of gamma rays for nuclear physics experiments. The particle accumulator ring (PAR), currently slated for decommissioning as a principal component of the APS injector complex, could likewise be converted into an IR light source capable of serving several users, or into a storage ring beam-dynamics research facility. If such a conversion were to be performed on the booster synchrotron, in particular, care must be taken not to have the operation of the booster-based light source conflict with top-up operation of 
the storage ring. A 5-second interruption in operations every 2 minutes should not be particularly onerous; however, more frequent interruptions could easily prove problematic.

\section{CONFLICTING GOALS}

There is a fundamental, if far-off, conflict between the goals of pushing towards greater development of the storage-ring performance and the transformation of the APS injector complex into a more general light-source resource. It is therefore reasonable to consider additional changes to the APS facility to support both branches of progress that would, in fact, add additional options for increased user support.

\section{LINAC-AUGMENTED LIGHT SOURCE}

A linac-augmented light source (LALS) uses a high-energy linear accelerator to greatly enhance the operational flexibility of the light source. The linac, for the purposes of this paper, is taken to be an L-band, superconducting, standing-wave cavity accelerator based on the TESLA project design. The primary linac has the same beam energy as the storage ring (in the case of the APS, $7 \mathrm{GeV}$ ), plus a small energy "overhead" to allow for redundancy, and an optional post-ring injection point secondary linac. For the purposes of this paper, the secondary linac is taken to have a maximum energy gain of $3 \mathrm{GeV}$.

\section{PRIMARY LINAC}

The primary linac would take over all top-up operational duties from the previous injector complex. It is expected that the injector complex would maintain the ability to fill the storage ring; however, with the addition of the primary linac, the preexisting injector complex could be reconfigured for use as separate light sources.

Table 1 lists the operating parameters of the primary linac when acting as a top-up driver for the storage ring. It should be noted that these do not represent particularly strenuous beam performance figures in any category, and the technology to achieve these numbers exists in operational form today.

As with most linacs, the per-bunch performance will be mainly limited by the performance of the injector. For this specification, the listed parameters should be readily met by most existing photoinjector systems. As a backup, a thermionic-cathode gun system, perhaps with some form of long-pulse laser-assisted emission, could also be used with a suitable beam gate to gate the number of bunches injected into the linac.

The emittance target was chosen so as to be able to inject into the storage ring with an unnormalized emittance of about $1 \mathrm{~nm}$. To date, the lowest emittance lattice installed at the APS has an unnormalized emittance of about $3 \mathrm{~nm}$; this number is expected to shrink, however, as smaller source sizes in the storage ring are pursued. The unnormalized emittance of the stored beam would need to fall below about $0.1 \mathrm{~nm}$ before the current state-of-the-art photoinjector performance is exceeded.

With a macropulse repetition rate of $100 \mathrm{~Hz}$, in principle the primary linac could provide for storage ring top-up operation with lifetimes as low as $10 \mathrm{~s}$. At that top-up rate, the maximum supportable storage-ring beam current would be $0.3 \mathrm{~A}$. If the lifetime were increased to 1 minute, the maximum supportable storage-ring beam current would be $1.8 \mathrm{~A}$. If the lifetime were fixed at 
what the booster synchrotron is currently capable of supporting, the primary linac could provide for a stored-beam current up to $15 \mathrm{~A}$.

It should be emphasized, however, that the limits presented here are purely those derived from the specifications on the injector systems; other factors, such as storage ring impedance and the availability of high-power x-ray optics, could easily impose considerably lower limits to the storedbeam current.

Table 1: Primary Linac Parameters

\begin{tabular}{||l|c|c||}
\hline PARAMETER & VALUE & UNITS \\
\hline General & & \\
\hline Total length & 600 & $\mathrm{~m}$ \\
\hline Cryomodules & 34 & \\
\hline Energy gain per module & 240 & $\mathrm{MeV}$ \\
\hline Total beam energy & 8.16 & $\mathrm{GeV}$ \\
\hline Average gradient & 13.6 & $\mathrm{MV} / \mathrm{m}$ \\
\hline RF system & & \\
\hline Operational frequency & 1.3 & $\mathrm{GHz}$ \\
\hline Average beam power & 800 & $\mathrm{~kW}$ \\
\hline Beam & & \\
\hline Charge per bunch & 1 & $\mathrm{nC}$ \\
\hline Bunches per macropulse & 1 & \\
\hline Normalized RMS emittance & 14 & $\mu \mathrm{m}$ \\
\hline RMS bunch length & & \\
\hline At injector & 10 & $\mathrm{ps}$ \\
\hline At exit of linac & $<1$ & $\mathrm{ps}$ \\
\hline Macropulse repetition rate & 100 & $\mathrm{~Hz}$ \\
\hline \multicolumn{2}{|l}{} \\
\hline
\end{tabular}

The addition of the primary linac system, therefore, serves two basic purposes. First, it allows for much shorter beam lifetimes and potentially higher stored-beam current than the existing injector complex. Second, it frees the remainder of the APS injector complex for conversion into additional light sources and related research facilities.

It should be noted again that the parameters of the primary linac system should be readily met, given currently operational, commercially available technology. Further, the system has several readily available upgrade paths, such as the addition of multiple bunches per macropulse, loweremittance injectors, or higher bunch-compression ratios.

\section{SECONDARY LINAC}

The secondary linac is an extension of the primary linac past the injection point for the storage ring, with a maximum energy gain of about $3 \mathrm{GeV}$; it is therefore somewhat less than half the length

\footnotetext{
* This figure assumes all $\mathrm{rf}$ modules operating at full power and on-crest (e.g., maximum energy gain). The stated value includes overhead both for redundancy and to allow off-crest operation for bunch compression.
} 
of the primary linac. Beam parameters would otherwise be identical; secondary linac parameters are shown in Table 2.

The purpose of the secondary linac is to leverage the existing primary linac as a beam source for another synchrotron radiation-based facility. The initial primary linac beam parameters can readily be upgraded to better emittance and shorter pulse durations; this transforms the primary linac into an ideal driver for a multi-user, multi-undulator SPPS or X-FEL facility.

The beam energy of the primary linac is effectively fixed by the requirement of providing beam to the storage ring at a high rate and at a fixed energy. Although in principle one could alter the beam energy in the primary linac shot-to-shot, in practice this is probably not an ideal solution; this problem, in a somewhat altered form, has already been encountered with the APS SASE-FEL user facility.

The secondary linac allows the beam energy from the primary linac to be adjusted by $+/-3 \mathrm{GeV}$, for a final energy range from $4-10 \mathrm{GeV}$. This capability provides a fast (potentially shot-to-shot) tuning range of a factor of 6 in wavelength, without undulator gap adjustment, and without any impact on the operation of the primary linac for storage-ring operation other than a potential reduction in the number of beam pulses available for top-up. A similar approach is currently being contemplated for the APS VUV-FEL user facility, to be accomplished by placing additional linac sections following the booster synchrotron injection point.

\section{Table 2: Secondary Linac Parameters}

\begin{tabular}{||l|c|c||}
\hline \hline PARAMETER & VALUE & UNITS \\
\hline General & & \\
\hline Total length & 240 & $\mathrm{~m}$ \\
\hline Cryomodules & 13 & \\
\hline Energy gain per module & 240 & $\mathrm{MeV}$ \\
\hline Total beam energy & $4-10$ & $\mathrm{GeV}$ \\
\hline Average gradient & 13.0 & $\mathrm{MV} / \mathrm{m}$ \\
\hline RF system & & \\
\hline Operational frequency & 1.3 & $\mathrm{GHz}$ \\
\hline Average beam powert & 300 & $\mathrm{~W}$ \\
\hline \multicolumn{2}{|l}{} \\
\hline
\end{tabular}

\section{A MODEL SYSTEM}

\section{SAMPLE LAYOUT}

An example layout as a whole is shown schematically in Figure 1. In this particular sketch, five undulator lines are shown, each with a length of $100 \mathrm{~m}$. This figure also indicates that the primary linac would inject into the storage ring at about the same point as the booster synchrotron. This might prove to be a convenience but not a necessity; the goal would be to minimize disruptions to existing beamlines installed on the storage rings. In any event, it is highly probable that the primary

† Indicates average beam power added by the secondary linac. 
and secondary linacs would be build significantly below the level of the existing APS storage ring; this would assist with radiation shielding as well as minimizing potential conflicts with existing and planned storage-ring beamlines. Similarly, while not as aesthetically pleasing in some respects, a long transfer line could be used to transport top-up shots from the linac to the APS storage ring; there is no overriding reason for them to be placed in this close a proximity, or in this particular orientation. Also, while keeping all undulator lines in the farm to one side of the secondary linac presents some potential advantages in terms of beam transport, there is no intrinsic reason why the undulator farm would have to be so constructed.

\section{POTENTIAL MODES OF OPERATION}

Using the layout shown in Figure 1 as a reference, and the performance figures shown in the tables above, several possible modes of operation can be considered.

Running in pure top-up mode, the primary linac would deliver beam to the APS storage ring at $100 \mathrm{~Hz}$, with potential minimum lifetime and maximum beam currents as calculated above. (See Appendix A for further details.) If the primary linac were to continue to run at $100 \mathrm{~Hz}$, but with every other beam pulse being diverted into the secondary linac, the effective top-up rate would be $50 \mathrm{~Hz}$, and the corresponding minimum lifetime would be $20 \mathrm{~s}$. The secondary linac would also receive beam at $50 \mathrm{~Hz}$, which, if split equally among the undulator farm beamlines, would result in an effective pulse repetition rate of $10 \mathrm{~Hz}$ at each undulator farm beamline.

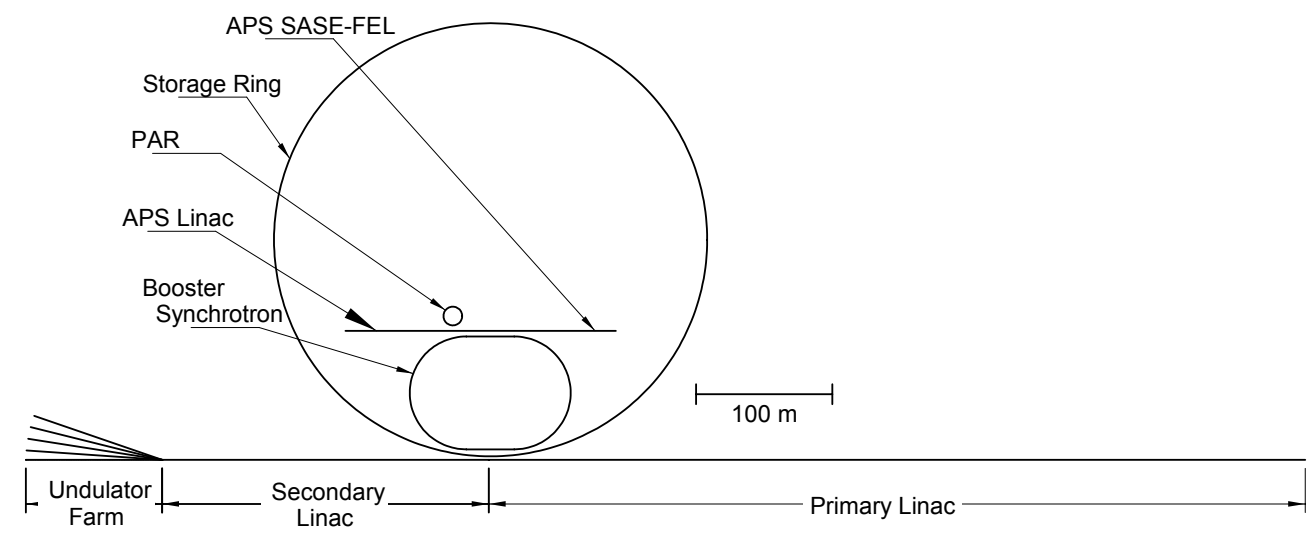

Figure 1: Schematic layout of a possible APS-based linac-augmented light source.

By varying the phasing of the individual accelerator modules within the secondary linac on a pulse-to-pulse basis, each incoming beam pulse can have an energy from $4-10 \mathrm{GeV}$. (Another option would be to vary the rf power pulse-to-pulse; however, in order to decelerate the beam, a phase change is also required.) Assuming that the beam pulse energies are distributed uniformly between 4 and $10 \mathrm{GeV}$, that each undulator branch line is made from identical undulators with 3.3 $\mathrm{cm}$ periods, and that $\mathrm{K}$ can be varied from 1 to 3.1, Table 3 lists the obtainable wavelength range 
from each undulator farm beamline. The tuning range of each branch line is shown graphically in Figure 2. Note that there is reasonable wavelength overlap from beamline to beamline.

Table 3: Undulator Farm Branch Beamline Minimum and Maximum Wavelengths for Given Beam Energies

\begin{tabular}{||c|c|c||}
\hline $\begin{array}{c}\text { BRANCH LINE } \\
\text { BEAM ENERGY } \\
{[\mathrm{GeV}]}\end{array}$ & $\begin{array}{c}\text { MINIMUM } \\
\text { WAVELENGTH } \\
(\mathrm{K}=1)[\AA]\end{array}$ & $\begin{array}{c}\text { MAXIMUM } \\
\text { WAVELENGTH } \\
(\mathrm{K}=3.1)[\AA]\end{array}$ \\
\hline 4.0 & 4.0 & 15.6 \\
\hline 5.5 & 2.1 & 8.3 \\
\hline 7.0 & 1.3 & 5.1 \\
\hline 8.5 & 0.89 & 3.5 \\
\hline 10.0 & 0.65 & 2.5 \\
\hline
\end{tabular}

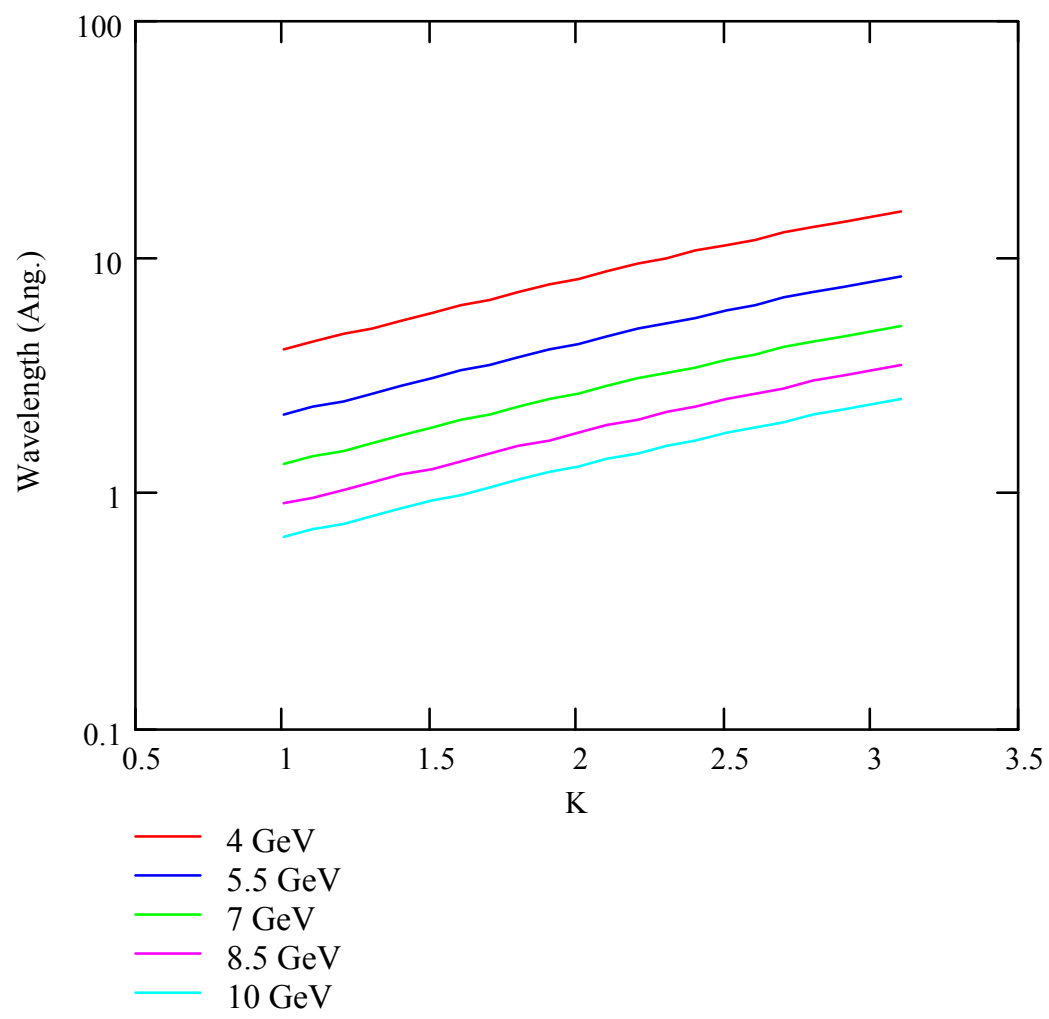

Figure 2: Undulator branch line tuning range, assuming uniform beam energy spacing and identical undulators.

\section{OTHER CONSIDERATIONS}

The installation of a superconducting linac to augment an existing light source could be planned as part of an overall machine upgrade package, potentially including the addition of superconducting, small-bore magnets and advanced insertion devices into the storage ring. A single cryo plant could 
then serve both the primary and secondary linacs as well as the storage ring requirements, lowering the average cost of both upgrades. Also, the existing APS linac could be replaced with superconducting $\mathrm{rf}$ modules and an improved injector for a relatively modest cost outlay; this would have the advantages of increasing the rep rate of beam delivered to the VUV FEL user facility and keeping all linac systems consistent within the facility.

The secondary linac need not be installed at the same time as the primary linac, nor would all proposed undulator farm beam tunnels have to be built at that time. However, any planning and construction work on the primary linac should include provisions for supporting the secondary linac and an extensive undulator farm.

\section{COST ESTIMATE}

Table 4 is a very rough cost estimate, based on the estimates for the proposed TESLA linear collider, and does not include manpower or contingency. It assumes that TESLA or a similar machine has already been constructed, and therefore the per-unit costs for the accelerator cavities and cryo tanks reflect the mass production price of these components.

Table 4: Cost Estimate Breakdown for LALS

\begin{tabular}{||l|c||}
\hline ITEM & $\begin{array}{c}\text { EST. COST } \\
{[\$ \mathrm{M}]}\end{array}$ \\
\hline Linac cryomodules (12 9-cell cavities per cryomodule) & 28 \\
\hline RF systems & 15 \\
\hline Injector complex & 10 \\
\hline $\begin{array}{l}\text { Civil engineering (incl. 5, 100-m undulator farm } \\
\text { tunnels) }\end{array}$ & 21 \\
\hline General infrastructure & 10 \\
\hline Controls and diagnostics systems & 10 \\
\hline Accelerator hardware (magnets, ps, vacuum, etc.) & 20 \\
\hline \multicolumn{2}{|l}{} \\
\hline Total estimate & 114 \\
\hline
\end{tabular}

The cost estimate also does not include the undulators themselves, nor the associated electron and photon beam diagnostics and controls, beamline optics, etc. The storage ring and SR user systems would require significant enhancement and upgrade to meet the performance levels allowed by a LALS linac; these are also not included in this estimate.

\section{CONCLUSIONS}

A linac-augmented light source relies on proven accelerator technology to greatly enhance the operational capabilities of a $3^{\text {rd }}$-generation storage ring. By adding a primary linac for on-energy injection into the storage ring, the existing injector complex can be recommissioned to support users at different wavelength regimes, e.g., UV to IR. By adding a secondary linac following the storage ring injection point, the facility can also support a series of $4^{\text {th }}$-generation light source techniques, notably single-pass free-electron lasers or short-pulse synchrotron sources. By using standard beam- 
energy control techniques in the secondary linac, a wide wavelength tuning range can be obtained even given identical undulators in the post-secondary linac undulator farm.

Unlike an energy-recovery linac, the LALS does not attempt to recapture the beam energy via a recirculation technique. Rather, it uses the inherently efficient nature of the storage ring to provide for an effective recirculation scheme, allowing the linac repetition rate to be relatively modest. Using an interleaving technique, the LALS still provides a reasonable repetition rate to the undulator farm users. One can also envision special operational modes, such as configuration of the storage ring into a one-orbit-and-dump mode, or a one-orbit-to-secondary-linac mode, in order to provide the storage ring users with short-pulse runs.

The estimate of the cost, while crude and not including items such as manpower and undulators, still indicates that such an upgrade may be very reasonable to consider as a future upgrade path for an existing $3^{\text {rd }}$-generation facility. In this fashion, a $3^{\text {rd }}$-generation light source can not only serve as a project support base for a $4^{\text {th }}$-generation facility, but also obtain greatly expanded operational capabilities for its existing and future user base. 


\section{APPENDIX A: STORED BEAM LIMIT CALCULATIONS}

This appendix does not consider the machine lattice, chamber, or other equipment modifications required to meet the discussed beam lifetimes and stored currents; rather, it uses the injector system parameters to determine ultimate limits.

The minimum allowable lifetime for a stored beam is given by

$$
\tau_{\min }=-\frac{1}{\mathrm{f}_{\mathrm{b}} \cdot \ln \left(\mathrm{C}_{1}\right)}
$$

where $\tau_{\min }$ is the minimum lifetime, $\mathrm{C}_{\mathrm{l}}$ is the desired beam current stability, and $\mathrm{f}_{\mathrm{b}}$ is the repetition rate of the injector. Note that this is independent of the beam charge. For instance, given a desired stability of $99.9 \%$ and a repetition rate of $2 \mathrm{~Hz}$, the minimum lifetime is approximately $500 \mathrm{~s}$. For a repetition rate of $100 \mathrm{~Hz}$, the minimum supportable lifetime is $10 \mathrm{~s}$.

The maximum supportable beam current in the ring, for a given lifetime $\tau_{\text {beam }}>\tau_{\min }$, is given by

$$
\mathrm{I}_{\max }=\frac{\mathrm{Q}_{\mathrm{b}} \mathrm{c}}{\mathrm{C}_{\text {ring }}\left(1-\mathrm{e}^{-1 / \mathrm{f}_{\mathrm{b}} \tau_{\text {beam }}}\right)},
$$

where $\mathrm{I}_{\max }$ is the maximum stored beam current, $\mathrm{Q}_{\mathrm{b}}$ is the charge injected per bunch, $\mathrm{c}$ is the speed of light, and $\mathrm{C}_{\text {ring }}$ is the storage ring circumference. If $\tau_{\text {beam }}=\tau_{\min }$, the equation reduces to

$$
\mathrm{I}_{\max }=\frac{\mathrm{Q}_{\mathrm{b}} \mathrm{c}}{\mathrm{C}_{\text {ring }}\left(1-\mathrm{C}_{1}\right)}
$$

The maximum top-up beam current, for a given stability level and beam lifetime, is limited by the maximum charge per bunch from the injector and is independent of the bunch rate $f_{b}$. Thus, when operating at minimum possible lifetimes for the stated charge stability level, a booster-based injection system has $\mathrm{I}_{\max }=1.5 \mathrm{~A}$ (assuming $5 \mathrm{nC}$ per bunch), while the full-energy linac, at $1 \mathrm{nC}$ per bunch, would be limited to $300 \mathrm{~mA}$. This does not take into account the dramatic difference in potential minimum lifetimes, however. For instance, if the lifetime was maintained at $500 \mathrm{~s}$ in both instances, $\mathrm{I}_{\max }=15 \mathrm{~A}$ for the full-energy linac vs. 1.5 A for the booster.

It should be emphasized that these are limits imposed by the operation of the injection system; actual operating limits in the ring might be much lower due to impedance effects, etc. 
APPENDIX B: ACRONYMS AND DEFINITIONS

LALS

Linac Augmented Light Source. Pronounced "Lawless" 


\section{REFERENCES}

1 P. Krejcik, "FEL Research and Development at the SLAC Short Pulse Photon Source," Proceedings of the 2002 Free-Electron Laser Conference, Argonne, IL, to be published. Presentation available online at http://www.aps.anl.gov/conferences/fel2002/talks/TU-O-08.pdf. 